\title{
Gaussian beam diffraction in free space
}

\author{
P. Berczyński, ${ }^{* 1}$ Yu. A. Kravtsov, ${ }^{2}$ \\ ${ }^{1}$ Institute of Physics, West Pomeranian University of Technology, Szczecin 70-310, Poland, \\ ${ }^{2}$ Institute of Physics, Maritime University of Szczecin, Szczecin 70-500, Poland
}

Received March 11, 2013; accepted March 21, 2013, published March 31, 2013

\begin{abstract}
In this paper a complex geometrical optics (CGO) method is applied for the Gaussian beam (GB) diffraction in free space. CGO reduces the diffraction description to solving ordinary differential equations for a beam amplitude and for a complex curvature of the wave front. We present analytical solutions for the GB width, wave front curvature and complex amplitude. Moreover, we discuss the influence of beam ellipticity on propagation in free space. Obtained CGO solutions are compared with the solutions of a diffraction theory, specifically from the Kirchhoff-Fresnel integral.
\end{abstract}

Complex geometrical optics (CGO) has two equivalent forms: the ray-based form, which deals with complex rays [1-6], that is with trajectories in a complex space, and the eikonal-based form, which uses complex eikonal instead of complex rays [6, 7]. A surprising feature of $\mathrm{CGO}$ is its ability to describe the Gaussian beam (GB) diffraction in both ray-based and eikonalbased approaches. Recently, an eikonal-based CGO method has been applied to describe GB evolution in inhomogeneous media [8, 9], nonlinear inhomogeneous fibres [10] and graded-index fibres [11]. It is shown in [11] that the eikonal-based CGO approach demonstrates high ability to describe GB evolution in graded-index optical fibres reducing hundred times the time of numerical calculations at comparable accuracy with a Crank-Nicolson scheme in the Beam Propagation Method (BPM).

For an axially symmetric wave beam propagating along the $\mathrm{z}$ axis in free space, CGO suggests a solution of the form

$$
u(r, z)=A \exp \left(i k_{0} \psi\right)=A(z) \exp \left[i k_{0}\left(B(z) r^{2} / 2-z\right)\right]
$$

where $\psi$ is the complex-valued eikonal, which in accordance with Eq. (1) has the form

$$
\psi=B(z) r^{2} / 2-z
$$

where $r=\sqrt{x^{2}+y^{2}}$ is the distance from the axis $z$ (radius in cylindrical symmetry) and parameter $B(z)$ is the complex curvature of the beam wave front. The eikonal equation in $(r, \mathrm{z})$ coordinates takes the form.

\footnotetext{
*E-mail: pawel.berczynski@zut.edu.pl
}

$$
\left(\frac{\partial \psi}{\partial r}\right)^{2}+\left(\frac{\partial \psi}{\partial z}\right)^{2}=1
$$

where $\varepsilon=1$. Substituting the complex eikonal from Eq. (2) into the eikonal equation in Eq. (3), we obtain a Riccati equation in the form

$$
\frac{d B}{d z}=B^{2}
$$

which has the following solution:

$$
B(z)=\frac{B(0)}{1-B(0) z} \cdot
$$

For the GB which has the initial width $\mathrm{w}(0)$ and with the initial wave curvature equal to zero $\kappa(0)=0$ the initial value of parameter $B$ is equal to $B(0)=i / k_{0} w^{2}(0)$ and as a result we obtain that

$$
B(z)=\frac{i / k_{0}(0)}{1-i z / k_{0} w^{2}(0)}=\frac{i / k_{0} w^{2}(0)}{1+z / i k_{0} w^{2}(0)} .
$$

The real and imaginary parts of this solution are equal to

$$
\begin{aligned}
& \operatorname{Re} B(z)=\frac{z}{k_{0}^{2} w^{4}(0)+z^{2}} \\
& \operatorname{Im} B(z)=\frac{k_{0} w^{2}(0)}{k_{0}^{2} w^{4}(0)+z^{2}} .
\end{aligned}
$$

As a result, the beam width and wave front curvature turn out to be

$$
\begin{gathered}
w=w(0) \sqrt{1+\left(\frac{z}{L_{D}}\right)^{2}}, \\
\kappa=\frac{z}{z^{2}+L_{D}^{2}} .
\end{gathered}
$$


where $L_{D}=k_{0} w^{2}(0)$ is the diffraction length. In the framework of a paraxial approximation, where $r$ is the small parameter, amplitude $\mathrm{A}=\mathrm{A}(\mathrm{z})$ satisfies the transport equation, which for the axially symmetric beam in $(r, \mathrm{z})$ coordinates takes the following form:

$$
\frac{d A^{2}}{d z} \frac{\partial \psi}{\partial z}+\left[\frac{1}{r} \frac{\partial}{\partial r}\left(r \frac{\partial \psi}{\partial r}\right)+\frac{\partial^{2} \psi}{\partial z^{2}}\right] A^{2}=0 \cdot
$$

In accordance with Eq. (2) we obtain that

$$
\frac{\partial \psi}{\partial z}=1, \frac{1}{r} \frac{\partial}{\partial r}\left(r \frac{\partial \psi}{\partial r}\right)=2 B
$$

and as a result, Eq. (10) reduces to an ordinary differential equation in the form:

$$
\frac{d A}{d z}+B(z) A=0
$$

As a result, the complex amplitude of cylindrically symmetric GB takes the form

$$
A(z)=A(0) \exp \left(-\int B(z) d z\right)
$$

where $A(0)$ is the initial amplitude. Substituting Eq. (5) into Eq. (13), we obtain the connection between the amplitude of GB and the complex wave front curvature in the form

$$
A(z)=\frac{A(0) B(z)}{B(0)}
$$

As a result, the complex amplitude is equal to

$$
A(z)=\frac{A(0)}{1+z / i k_{0} w^{2}(0)}
$$

Let us compare the obtained CGO results presented in Eq. (6) and Eq. (15) for GB diffraction in free space with the solutions of a diffraction theory in the Fresnel approximation for which the diffraction integral has the form

$$
\begin{aligned}
& \mathrm{E}(x, y, z)=\frac{i}{\lambda z} e^{-i k_{0} z} \int_{-\infty}^{+\infty} \int_{-\infty} \mathrm{E}_{0}\left(x^{\prime}, y^{\prime}\right), \\
& \exp \left\{\frac{i k_{0}}{2 z}\left[\left(x-x^{\prime}\right)^{2}+\left(y-y^{\prime}\right)^{2}\right]\right\} d x^{\prime} d y^{\prime}
\end{aligned}
$$

where $\left(x^{\prime}, y^{\prime}\right)$ are the coordinates within the plane of the screen, $(x, y)$ are the coordinates in the observation plane, $\mathrm{E}(x, y, z)$ is the envelope of the field in othe bservation plane, $\mathrm{E}_{0}\left(x^{\prime}, y^{\prime}\right)$ is the field envelope in the plane of the screen with the aperture and $z$ is the distance between the screen plane and the observation plane. The field envelope $\mathrm{E}_{0}\left(r^{\prime}\right)$ in the plane of the screen with the aperture has the form of GB

$$
\mathrm{E}_{0}\left(r^{\prime}\right)=E_{0} \exp \left(-r^{\prime 2} / 2 w_{0}^{2}\right)
$$

we obtain on the observation plane that diffraction field has the form

$$
\mathrm{E}(r, z)=\frac{\mathrm{E}_{0} \exp \left(-i k_{0} z\right)}{1+z / i k_{0} w_{0}^{2}} \exp \left[-\frac{r^{2} / 2 w_{0}^{2}}{1+z / i k_{0} w_{0}^{2}}\right]
$$

One can notice that the above solution is in total agreement with the CGO wave field in the form $u(r, z)=A \exp \left(i k_{0} \psi\right)$, where complex amplitude $A$ for GB propagating in free space is presented in Eq. (15) and complex eikonal $\psi$ is defined in Eq. (2) together with the complex wave front curvature in Eq. (6). Thus, for Gaussian beam propagating and diffracting in free space the CGO method gives the same result as obtained within the Fresnel approximation to the Kirchoff integral, taking into account that wave function $u(r, z)$ plays the same role as field envelope $\mathrm{E}(r, z)$ and quantity $A(0)$ in Eq. (15) is equivalent with parameter $\mathrm{E}_{0}$ in Eq. (17). Moreover, the CGO method supplies this solution in a much simpler way. The diffraction of GB with a circular cross section can be easily generalized for the case of an elliptical cross-section beam in the framework of the CGO method. In the framework of the CGO method an elliptical GB has the form

$$
u=A_{0} \sqrt{\frac{w_{10} w_{20}}{w_{1} w_{2}}} \exp \left(i k B_{1} x^{2} / 2+i k B_{2} y^{2} / 2\right)
$$

In above Eq. (19) complex parameters $B_{1}$ and $B_{2}$ are connected with principle widths and wave front curvatures 
by the relations $\quad B_{1}=\kappa_{1}+i / k_{0} w_{1}^{2} \quad$ and $B_{2}=\kappa_{2}+i / k_{0} w_{2}^{2}$. Riccati equations have the form

$$
\frac{d B_{i}}{d z}=B_{i}^{2}
$$

These equations can be readily integrated, obtaining the following solutions:

$$
B_{i}=\frac{B_{i 0}}{1+B_{i 0} z},
$$

where $B_{i 0} \equiv B_{i}(0)$. By substituting Eq. (21) into equation for complex amplitude $A$ :

$$
\frac{d A}{d z}+\frac{1}{2} \operatorname{Tr} B_{i j} A=0
$$

we obtain the equation

$$
\frac{d A}{d z}+\frac{1}{2}\left(\frac{B_{10}}{1+B_{10} z}+\frac{B_{20}}{1+B_{20} z}\right) A=0,
$$

where $\varepsilon_{c}=1$ and $\psi_{c}=\int \varepsilon_{c} d z=z$. The Eq. (23) next yields

$$
A=\frac{A_{0}}{\sqrt{\left(1+B_{10} z\right)\left(1+B_{20} z\right)}}=A_{0} \sqrt{\frac{B_{1} B_{2}}{B_{10} B_{20}}} .
$$

Gaussian beam principle widths and wave front curvatures have the form

$$
\kappa_{i}=\frac{1}{z\left[1+\left(\frac{L_{R i}}{z}\right)^{2}\right]}, w_{i}=w_{i 0} \sqrt{1+\left(\frac{z}{L_{R i}}\right)^{2}}
$$

where $L_{R i}=k_{0} w_{i 0}^{2}$ is the Rayleigh distance, corresponding to the initial width $w_{i 0} \equiv w_{i}(0)$. Equations (25) demonstrate a typical behaviour for GB diffraction in free space. In the near zone, $z<<L_{R i}$, GB widths are approximately constant, whereas the curvatures of the wave front increase:

$$
w_{i} \approx w_{i 0}=\text { const }, \kappa_{i}=\frac{z}{L_{R i}^{2}}
$$

In the far zone, $z>>L_{R i}$, the beam widths increase and the curvatures of the wave front decrease:

$$
\left.w_{i} \approx \frac{w_{i 0}}{L_{R i}} z, \quad Y_{i} 78\right)_{z}
$$

In the near zone (with the plane initial phase front) the absolute value of complex amplitude $A$ remains almost constant:

$$
\left.|A| \approx \mid A_{0}^{(79}\right) \text { const }
$$

while in the far zone

$$
|A| \approx\left|A_{0}\right| \frac{k_{0} w_{10} w_{20}}{z}=\left|A_{0}\right| \sqrt{\frac{w_{10} w_{20}}{w_{1} w_{2}}} .
$$

Thus, Eq. (29) agrees with the conservation of energy flux through a beam cross-section.

\section{References}

[1] Yu.A. Kravtsov, Radiophys. Quant. Electronics 10, 719 (1967)

[2] J.B. Keller, W. Streifer, J. Opt. Soc. Am. 61, 40 (1971).

[3] G.A. Deschamps, Electron. Lett. 7(23), 684 (1971).

[4] Yu.A. Kravtsov, G.W. Forbes, A.A. Asatryan, Progress in Optics 39, 3 (1999).

[5] S.J. Chapman, J.M. Lawry, J.R. Ockendon, R.H. Tew, SIAM Rev. 41, 417 (1999).

[6] Yu.A. Kravtsov, P. Berczynski, Stud. Geophys. Geod. 51(1), 1 (2007).

[7] Yu.A. Kravtsov N.Y. Zhu. Theory of diffraction: Heuristic Approaches (Alpha Science International, ISBN 1842653725, 2009).

[8] P. Berczynski, Yu.A. Kravtsov, Phys. Lett. A, 331(3-4), 265 (2004).

[9] P. Berczynski, K.Yu. Bliokh, Yu.A. Kravtsov, A. Stateczny, J. Opt. Soc. Am. A 23(6), 1442 (2006).

[10] P. Berczyński, Yu. Kravtsov, Phot. Lett. Poland, 4(1), 26 (2012).

[11] P. Berczynski, Yu.A. Kravtsov, G. Zeglinski, Opt. Appl. 40(3), 705 (2010). 